\title{
An STM Investigation on a Metal Induced Structural Evolution of an $m$-Substituted Bipyridine Derivative on Highly Oriented Pyrolytic Graphite
}

\author{
Yi Wang, ${ }^{a, b}$ Liang Luo, ${ }^{b}$ and Qunhui Yuan ${ }^{*, a, b}$ \\ ${ }^{a}$ School of Materials Science and Engineering, Harbin Institute of Technology, Shenzhen, Guangdong 518055, China \\ ${ }^{b}$ Laboratory of Environmental Science and Technology, Xinjiang Technical Institute of Physics and Chemistry, \\ Chinese Academy of Sciences, Urumqi, Xinjiang 830011, China
}

\begin{abstract}
Due to an increasing demand for miniaturized electronics, on-surface molecular assembly has progressed rapidly for its advantage of fabricating highly organized patterns at nanoscale. In this regard, on-surface metal-organic architectures of bipyridine derivates have been invoked owing to moderate strength and flexible direction of metal-ligand interaction. Despite intensive researches on self-assembled monolayers of $p$-substituted bipyridine derivates and their metal complexes, few reports have addressed the structural evolution of $m$-substituted ones. In this paper, a 4,4'-dinonyl-2,2'-bipyridine with two substitutional alkyl chains at $m$-positions was employed as a ligand for construction of metallosupramolecular architectures with zinc and copper centers via metal coordination, respectively. A microscopic observation on metal directed structural transformations of 4,4'-dinonyl-2,2'-bipyridine arrays, together with merits from previous related researches, reveals more understanding on the rational design of on-surface bipyridine derivates based metal-organic architectures.
\end{abstract}

Keywords surface confined self-assembly, metal-organic architectures, bipyridine derivates, structural evolution, in situ coordination

\section{Introduction}

Owing to the limitation of "top-down" strategy for obtaining designed patterns at nanoscale, "bottom-up" nanofabrication has been considered as a possible alternative route in the fields of electronics, sensors and optical devices. ${ }^{[1-4]}$ Among all patterns prepared via "bottom-up" routes, the surface confined self-assemblies constituted by molecules have attracted intensive interests, contributable to their potential applications in engineering functional surfaces. ${ }^{[4-10]}$ To achieve appropriate functionality of the self-assembly decorated surface, the precise arrangement of block molecules on substrate is highly desired, considering the intimate relationship between the low-dimensional surface confined nanopatterns and the performance of the obtained surfaces. ${ }^{[1,9,11-20]}$ In this case, the design concept based on the on-surface metal-organic coordination is handy, for its capacity in obtaining the surface confined nanostructures with regular patterns.

Bipyridine derivate (bpy), as a good candidate for fabrication of on-surface metal-organic architectures via coordination, has lately drawn great attention in "bottom-up" nanofabrication relying on moderate rigidity and directionality of coordination bonds. ${ }^{[10,21-25]}$ To date, many efforts have been devoted to improving understanding on the adjustment of bpy assembly on surface or at interface. For example, De Feyter et al. ${ }^{[26,27]}$ reported a series of packing structures and metal induced motif transformations of bpys on highly oriented pyrolytic graphite (HOPG). By tailoring the substitutional groups on the $p$-positions in bpys, the intermolecular distances between bpys could be tuned, thus leading to varied coordinating capabilities of the surface confined bpys. They also found that the structural symmetry of bpys and the types of metal centers would also affect the packing patterns of bpys on surface. Kikkawa et al. ${ }^{[28-31]}$ studied systematically the odd-even effect and the coordination induced on-surface motif transformations of bpys, in which two sets of alkoxyphenyl ethynyl groups were linked to the bipyridine unit at the $p$-positions on both sides. By tuning the odd-even number of carbon atoms in the side chain, the single/double chain substitution, and the alkyl chain position in the alkoxyphenyl group, successful modification on the assembled arrays of bpys had been achieved. ${ }^{[28,30]}$ They also demonstrated that the metal coordination would cause further change of absorbed bpy arrays. For example, the odd-even effect would be quenched upon metal coordination, leading to a convergence of lamellar structure in bpy arrays. ${ }^{[29]}$ These investigations indicate that the manipulation of side groups and metal centers may provide a chance to get the pre-conceived motifs of bpys. Nevertheless, the relationship between molecular structures and on-surface packing patterns of bpys is not quite clear yet, especially in regard to the effect of substitution positions of side chains on the evolution of metal induced on-surface structures of bpys. For instance, our previous study showed that a 4,4'-ditetradecyl-2,2'-bipyridine (14-bpy) with $m$-substituted tetradecyl group at both sides would only form a non-interdigtated packing array on $\mathrm{HOPG},{ }^{[32]}$ which is quite different from the packing patterns of its analogues reported by Kikkawa et al. and De Feyter et al. ${ }^{[27,28]}$ This finding implies that the different substituted position in bpys may bring about different mechanism of packing pattern changes upon metal complexation. However, an investigation on the effect of varied substitutional groups at the $m$-positions in bpys on their coordinated motifs is rare.

In this extended microscopic study, an $m$-substituted bpy, 4,4'-dinonyl-2,2'-bipyridine (9-bpy), was used as a ligand and a metal induced motif transition of 9-bpy was studied by tailoring metal centers. It is worth noting that the 9-bpy used in this study bears a similar

* E-mail: yuanqunhui@hit.edu.cn; Tel.: 0086-0755-86244014 Received April 18, 2017; accepted May 16, 2017. 
molecular structure with our previously reported $14-\mathrm{bpy},{ }^{[32]}$ thus enables us to carry out a comparison study on the effect of side chain length and the possible odd-even effect on the coordination motifs of the $m$-substituted bpys. On the basis of STM observation, a chain length and metal center dependent coordination motif diversity of bpy was revealed, which provides insights into the design of surface confined metal-organic architectures.

\section{Experimental}

9-Bpy (97\%, Alfa) and ethanol (99.97\%, J.T. Baker) were used as received. On-surface assembly of 9-bpy was prepared by drop casting its ethanol solution $\left(\sim 10^{-4} \mathrm{~mol} / \mathrm{L}\right)$ onto a freshly cleaved HOPG surface (ZYB grade, Bruker), and then was checked with STM after drying in air for several minutes. To investigate coordination between metal ions and 9-bpy, a drop of $\mathrm{Zn}$ (II)/Cu(II) ethanol solution $\left(\sim 10^{-4}\right.$ $\mathrm{mol} / \mathrm{L}$ ) was added onto HOPG at the same location of the initial droplet containing 9-bpy, after 9-bpy/HOPG had been set in air for $\sim 5$ min. To capture the spontaneous structural change in the pre-adsorbed bpy array upon metal addition, STM observation was carried out $\sim 5$ min after the addition of metals.

The STM investigation was performed with a Nanoscope IIId scanning probe microscopy (Bruker, USA) system under ambient conditions. Mechanically cut $\mathrm{Pt} / \mathrm{Ir}$ wires $(90 / 10)$ were used as the STM tips. All of the STM images were obtained under the constant current mode. The operational parameters used for each presented STM images are provided in the corresponding figure captions.

\section{Results and Discussion}

\section{Packing pattern of 9-bpy on HOPG}

The chemical and optimized structures of 9-bpy were shown in Figure 1 . Figures $2 \mathrm{~A}$ and $2 \mathrm{~B}$ showed typical large scale and high resolution STM images of 9-bpy array on the HOPG surface, respectively. The contrast of bright and dark stripes in Figure 2B indicated bipyridine moieties and aliphatic chains in 9-bpy. The distance between two adjacent 9-bpys within one lamella is about $0.7 \mathrm{~nm}$ (defined as M1), indicating the 9-bpy absorbed on surface with its bipyridine moiety parallel to the graphite plane. ${ }^{[26,28]}$ The length between adjacent lamellas is approximately $1.9 \mathrm{~nm}$ (defined as L1), implying the alkyl chains of 9-bpy are extended in an almost non-interdigitated fashion. ${ }^{[27,31]}$ Based on these data, a corresponding packing model of 9-bpy was proposed in Figure 2C.

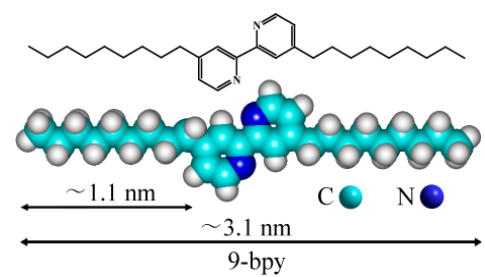

Figure 1 Chemical and optimized structures of 9-bpy.
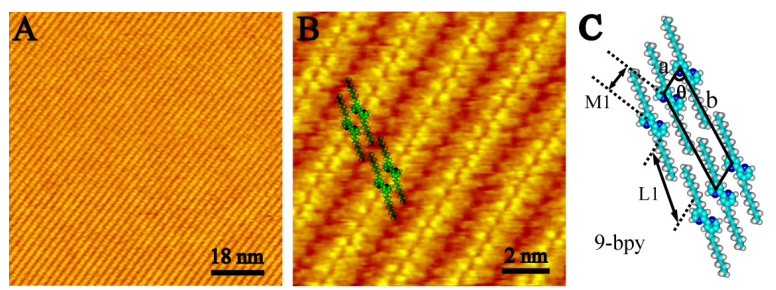

Figure 2 (A) A typical large-scale STM image of 9-bpy array on HOPG $(I=300 \mathrm{pA}, V=800 \mathrm{mV})$; (B) A high-resolution STM image of (A) $(I=341 \mathrm{pA}, V=780 \mathrm{mV})$; (C) Adsorption model.
It is worth noting that 14-bpy also adopts a similar flat-lying orientation as that of 9-bpy. ${ }^{[32]}$ Unlike the tail-core-tail linear-type arrangement of the alkyl chains in 9-bpy, the alkyl chains in 14-bpy bend away from the bipyridine core to take a more close-packed alignment, as also illustrated by the experimental parameters listed in Table 1. This different orientation is likely caused by the varied matches between the adsorbates and graphite plane, as well as the varied adsorption energies of 9-bpy and 14-bpy on surface. Similar phenomena have also been observed in arrays of other alkane derivatives, in which the alkyl chains take different angles to fulfill the formation of hydrogen bonds. ${ }^{[9,33-36]}$

Table 1 Adsorption parameters of $m$-bpys and their complexes arrays $^{a}$

\begin{tabular}{cccccc}
\hline Adsorbate & $a / \mathrm{nm}$ & $b / \mathrm{nm}$ & $\theta /\left({ }^{\circ}\right)$ & $M / \mathrm{nm}$ & $L / \mathrm{nm}$ \\
\hline 9-bpy & 0.8 & 2.8 & 60 & 0.7 & 1.9 \\
14-bpy & 0.8 & 4.3 & 53 & 0.7 & 3.4 \\
9-bpy/Zn(II) & 0.8 & 2.4 & 63 & 0.7 & 1.5 \\
14-bpy/Zn(II) & 0.5 & 3.5 & 82 & 0.5 & 2.9 \\
9-bpy/Cu(II) & 0.8 & 2.2 & 82 & 0.7 & 1.1 \\
14-bpy/Cu(II) & 0.5 & 2.7 & 81 & 0.5 & 1.8 \\
\hline
\end{tabular}

${ }^{a}$ The adsorption parameters of 14-bpy and 14-bpy/metal arrays are cited from Ref. [32]. Error bars: distance $\pm 0.1 \mathrm{~nm}$; angle $\pm 2^{\circ}$.

In addition, the packing patterns of $m$-substituted 9-bpy and 14-bpy are similar to that of $p$-substituted bpy reported by De feyter et al., ${ }^{[26,27]}$ except for only one type of adsorption structure was observed for 9-bpy and 14-bpy arrays. This may be due to the different structural symmetry of $m$ - and $p$-substituted bpys. However, the pack patterns of 9-bpy and 14-bpy are significantly different from their counterparts reported by Kikkawa et al. ${ }^{[28,31]}$ in which the aliphatic chains of bpys are observed to be interdigitated. This indicates that the existence of two additional aromatic units in bpys would also exert great influence on controlling their self-assembly structures.

\section{Structural evolution of 9-bpy induced by metal coordination}

Zinc and copper ions are used to check the possible structural evolution of 9-bpy upon metal complexation. After in situ addition of $\mathrm{Zn}(\mathrm{II})$ on the pre-adsorbed 9-bpy array, a new packing feature with few irregular defects was observed (Figure 3A). The distance between two successive bright protrusions is about $0.7 \mathrm{~nm}$ (defined as M2), implying the bipyridine moiety keeps its flat-lying orientation after metal complexation. The coordinated sites for $\mathrm{Zn}(\mathrm{II})$ are likely to locate in the middle of two neighboring bipyridine moieties, as has also been observed and interpreted by De Feyter et al. ${ }^{[26,27]}$ and Kikkawa et al. ${ }^{[28-31]}$ The distance between the adjacent lamellas shrunk to $1.5 \mathrm{~nm}$ (defined as L2), suggesting the alkyl chains in the formed 9-bpy/Zn(II) complex were partly interdigitated, as detailed in the adsorption model in Figure 3B.

The in situ coordination of the pre-adsorbed 9-bpy array with $\mathrm{Cu}$ (II) has also been carried out for comparison. As shown in Figure $3 \mathrm{C}$, the intermolecular distance within one lamella is about $0.7 \mathrm{~nm}$ (defined as M3), which is comparable with the values of M1 and M2, whereas the distance between the adjacent bright lamellas (L3) decreased from $\sim 1.9 \mathrm{~nm}$ to $\sim 1.1 \mathrm{~nm}$ after complexation. These parameters imply that the bipyridine moiety in the 9-bpy/Cu(II) complex keeps its flat-lying orientation, while the alkyl chains are fully inter digitated with each other. Besides, the angle $(\theta)$ between $a$ and $b$ directions remarkably increased from $\sim 60^{\circ}$ to $\sim 82^{\circ}$, owing to the presumable requirement on the adjustment of surface adsorption energy of 9-bpy upon $\mathrm{Cu}(\mathrm{II})$ complexation. 


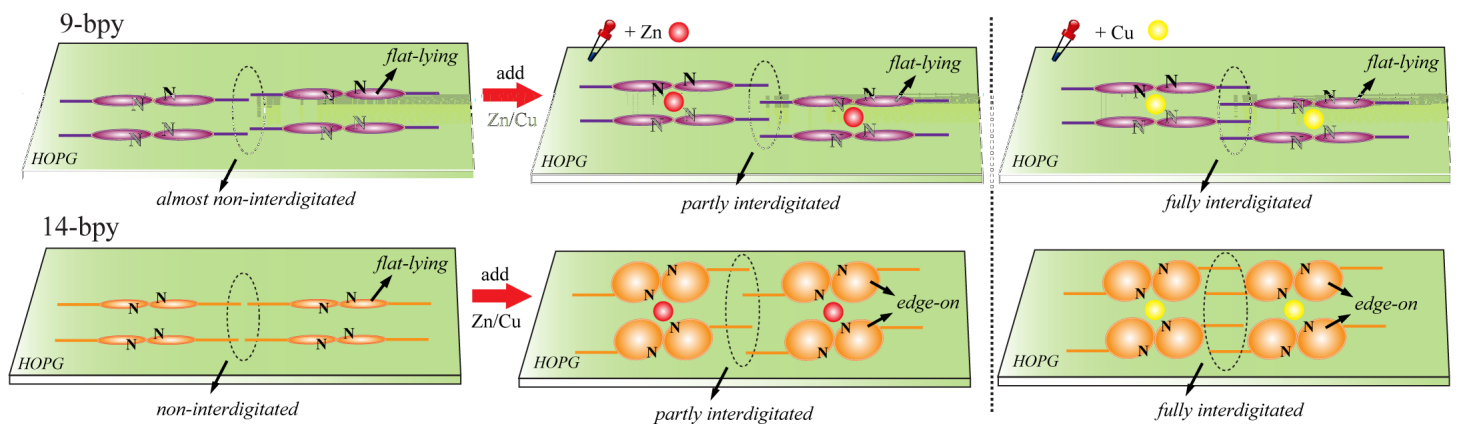

Figure 4 Packing patterns of 9-bpy, 14-bpy and their metal complexes arrays on HOPG.

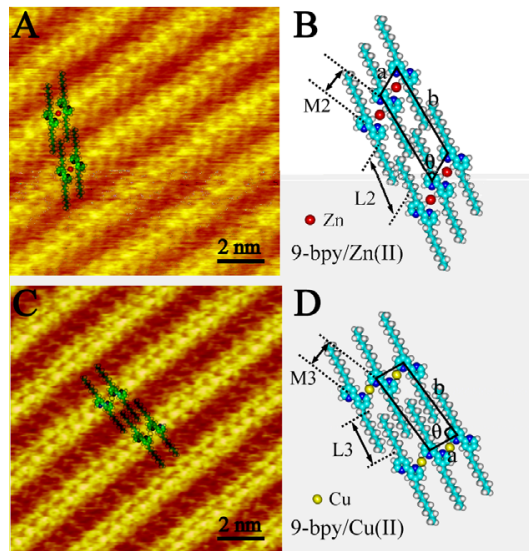

Figure 3 (A) and (C): High-resolution STM images of 9-bpy/Zn(II) and 9-bpy $/ \mathrm{Cu}(\mathrm{II})$ complexes arrays after in situ metal coordination $(I=343 \mathrm{pA}, V=800 \mathrm{mV}$ and $I=286 \mathrm{pA}, V=800 \mathrm{mV})$. (B) and (D): Adsorption models for (A) and (C).

Encouraged by the odd-even chain length effect on the packing patterns of bpys reported by Kikkawa et al., ${ }^{[29]}$ we also compared the present metal-induced structural change of 9-bpy with that of the 14-bpy. It is noted that 9-bpy and 14-bpy shared similar flat-lying orientations in their bare molecular arrays, whereas 14-bpy changes to an oblique alignment and results in a dramatically decreased space between two neighboring 14-bpys upon metal coordination (Figure 4 and Table 1). Such metal-induced distortion of adsorbates have also been discussed by Barth et al. ${ }^{[6]}$ and Yuan et al. ${ }^{[37]}$ Due to the dependence between the adsorbate orientation and the subtle balance of intermolecular/molecular-substrate interactions, ${ }^{[7,8,30,38]}$ it is reasonable to assume that the different orientations of $\pi$-conjugated units in bpy/metal may result from the different sizes of 9-bpy and 14-bpy molecules, since the varied molecular dimensions may lead to different packing symmetries and lattice matches. ${ }^{[39,40]}$

The above observation and comparison also demonstrated that $\mathrm{Zn}$ (II) and $\mathrm{Cu}(\mathrm{II})$ have different capacities on tuning the on-surface structures of bpys, implying that the type of metal centers plays an important role in adjusting the coordination motifs of bipyridine derivatives.

\section{Conclusions}

In summary, an on-surface monolayer of 4,4'-dinonyl-2,2'-bipyridine was constructed to investigate the metal induced structural evolution of $m$-substituted bipyridine derivates. The $m$-substituted 9-bpy showed a flat-lying orientation with its alkyl chains fully extended on HOPG after adsorption. Upon in situ coordination with metal ions, 9-bpy keeps the flat-lying orientation despite the structural changes of the arrays. The alignment of the $m$-substituted chains in 9-bpy showed a metal-dependent packing feature. A comparison between the metal-organic architectures of 9-bpy and previously studied 14-bpy demonstrated that the substitutional chain length has a distinctive effect on the metal induced architectures of the $m$-substituted bpys, thus may very helpful for the adjustment of self-assembled coordination motifs of bipyridine derivatives.

\section{Acknowledgement}

The authors acknowledge the support from the National Natural Science Foundation of China (No. 21473247) and the Scientific Research Foundation for the New Teacher of Harbin Institute of Technology (Shenzhen).

\section{References}

[1] Gazit, E. Chem. Soc. Rev. 2007, 36, 1263.

[2] Barth, J. V. Surf. Sci. 2009, 603, 1533.

[3] Cronin, L. Annu. Rep. Prog. Chem., Sect. A 2006, 102, 353.

[4] Long, J. R.; Yaghi, O. M. Chem. Soc. Rev. 2009, 38, 1213.

[5] Mali, K. S.; Adisoejoso, J.; Ghijsens, E.; De Cat, I.; De Feyter, S. Acc. Chem. Res. 2012, 45, 1309

[6] Lin, N.; Stepanow, S.; Ruben, M.; Barth, J. V. Top. Curr. Chem. 2009, $287,1$.

[7] De Feyter, S.; De Schryver, F. C. J. Phys. Chem. B 2005, 109, 4290.

[8] De Feyter, S.; De Schryver, F. C. Chem. Soc. Rev. 2003, 32, 139.

[9] Donna, M. C.; Bhawani, V.; A. Flynn, G. W.; Black, A.; Whitesides, G. M. J. Phys. Chem. 1996, 100, 13747.

[10] Bebensee, F.; Svane, K.; Bombis, C.; Masini, F.; Klyatskaya, S.; Besenbacher, F.; Ruben, M.; Hammer, B.; Linderoth, T. R. Angew. Chem., Int. Ed. 2014, 53, 12955

[11] Elemans, J. A. A. W.; De Feyter, S. Soft Matter 2009, 5, 721.

[12] Giancarlo, L. C.; Flynn, G. W. Acc. Chem. Res. 2000, 33, 491.

[13] Lei, S. B; Wang, C.; Yin, S. X; Wang, H. N.; Xi, F.; Liu, H. W.; Xu, B.; Wan, L. J; Bai, C. L. J. Phys. Chem. B 2001, 105, 10838.

[14] Kervella, Y.; Shilova, E.; Latil, S.; Jousselme, B.; Silly, F. Langmuir 2015, 31, 13420.

[15] Afsari, S.; Li, Z. H.; Borguet, E. ChemPhysChem 2016, 17, 3385.

[16] He, Y.; Ye, T.; Borguet, E. J. Phys. Chem. B 2002, 106, 11264.

[17] Gao, A. M.; Miao, X. R.; Liu, J.; Zhao, P.; Huang, J. W.; Deng, W. L. ChemPhysChem 2010, 11, 1951.

[18] Miao, X. R.; Chen, C. M.; Zhou, J.; Deng, W. L. Appl. Surf. Sci. 2010, $256,4647$.

[19] Zhang, X.; Li, S. S.; Chen, T.; Wang, D.; Wan, L. J. Nano 2012, 7, 1230001.

[20] Liu, J.; Zhang, X.; Yan, H. J.; Wang, D.; Wang, J. Y.; Pei, J.; Wan. L. J. Langmuir 2010, 26, 8195.

[21] Zheng, X. Y.; Ye, L. Q.; Wen, Y. H. J. Mol. Struct. 2011, 987, 132.

[22] Kaes, C.; Hosseini, M. W.; Rickard, C. E. F.; Skelton, B. W.; White, A. H. Angew. Chem., Int. Ed. 1998, 37, 920.

[23] Visser, J.; Katsonis, N.; Vicario, J.; Feringa, B. L. Langmuir 2009, 25, 
5980.

[24] Semenov, A.; Spatz, J. P.; Möller, M.; Lehn, J. M.; Sell, B.; Schubert, D.; Weidl, C. H.; Schubert, U. S. Angew. Chem., Int. Ed. 1999, 38, 2547.

[25] Yuan, Q. H.; Wan, L. J. Chem. Eur. J. 2006, 12, 2808.

[26] Abdel-Mottaleb, M. M. S.; Schuurmans, N.; De Feyter, S.; Van Esch, J.; Feringa, B. L.; De Schryver, F. C. Chem. Commun. 2002, 1894.

[27] De Feyter, S.; Abdel-Mottaleb, M. M. S.; Schuurmans, N.; Verkuijl, B. J. V.; Van Esch, J. H.; Feringa, B. L.; De Schryver, F. C. Chem. Eur. J. 2004, $10,1124$.

[28] Kikkawa, Y.; Koyama, E.; Tsuzuki, S.; Fujiwara, K.; Miyake, K.; Tokuhisa, H.; Kanesato, M. Surf. Sci. 2007, 601, 2520.

[29] Kikkawa, Y.; Koyama, E.; Tsuzuki, S.; Fujiwara, K.; Miyake, K.; Tokuhisa, H.; Kanesato, M. Chem. Commun. 2007, 1343.

[30] Kikkawa, Y.; Koyama, E.; Tsuzuki, S.; Fujiwara, K.; Kanesato, M. Langmuir 2009, 26, 3376.

[31] Kikkawa, Y.; Koyama, E.; Aoyagi, M.; Schneider, N.; Takahashi, M.; Fujiwara, K.; Kanesato, M. Supramol. Chem. 2011, 23, 9.

[32] Wang, Y.; Yuan, Q. H.; Xu, H. B.; Zhu, X. F.; Gan, W. J. Chem. Phys.
2016, 145, 034701.

[33] Yin, S. X.; Wang, C.; Xu, Q. M.; Lei, S. B.; Wan, L. J.; Bai, C. L. Chem Phys. Lett. 2001, 348, 321.

[34] Bléger, D.; Bocheux, A.; Kreher, D.; Mathevet, F.; Attias, A. J.; Metgé, G.; Douillard, L.; Fiorinidebuisschert, C.; Charra, F. Nanoscale 2013, 5, 1452.

[35] Yokoyama, S.; Hirose, T.; Matsuda, K. Chemistry 2015, 21, 13569.

[36] Hu, Y.; Miao, K.; Zha, B.; Xu, L.; Miao, X. R.; Deng, W. L. Phys. Chem. Chem. Phys. 2015, 18, 624.

[37] Xu, H. B.; Wang, H. H.; Zhou, S. H.; Xiao, L. L.; Yan, Y.; Yuan, Q. H. RSC Adv. 2015, 5, 106061.

[38] Diao, Y. X.; Han, M. J.; Wan, L. J.; Itaya, K.; Uchida, T.; Miyake, H.; Yamakata, A.; Osawa, M. Langmuir 2006, 22, 3640.

[39] Yuan, Q. H.; Wan, L. J.; Jude, H.; Stang, P. J. J. Am. Chem. Soc. 2005, 127,16279

[40] Wu, P.; Zeng, Q.; Xu, S.; Wang, C.; Yin, S.; Bai, C. L. ChemPhysChem 2001, 2, 750 . 